\title{
Conformal holomorphically projective mappings satisfying a certain initial condition
}

Hana Chudá and Mohsen Shiha 


\title{
CONFORMAL HOLOMORPHICALLY PROJECTIVE MAPPINGS SATISFYING A CERTAIN INITIAL CONDITION
}

\author{
HANA CHUDÁ AND MOHSEN SHIHA
}

\begin{abstract}
In this paper we study conformal holomorphically projective mappings between conformal $e$-Kähler manifolds $K_{n}=(M, g, F)$ and $\bar{K}_{n}=(\bar{M}, \bar{g}, \bar{F})$, i. e. diffeomorphisms $f: M \rightarrow$ $\bar{M}$ satisfying $f=f_{1} \circ f_{2} \circ f_{3}$, where $f_{1}, f_{3}$ are conformal mappings and $f_{2}$ is a holomorphically projective mapping between $e$-Kähler manifolds (i. e. Kähler, pseudo-Kähler and hyperbolic Kähler manifolds).

Suppose that the initial condition $f^{*} \bar{g}=k \cdot g$ is satisfied at a point $x_{0} \in M$ and that at this point the Weyl conformal tensor satisfies a certain inequality. We prove that the mapping $f$ is then necessarily conformal.
\end{abstract}

2000 Mathematics Subject Classification: 53B20; 53B30; 53C21

Keywords: conformal mappings, holomorphically projective mappings, conformal holomorphically projective mappings, conformal $e$-Kähler manifolds, initial conditions

\section{INTRODUCTION}

One may say that the pioneering work in conformal and projective geometry was done by H. Weyl [19] and T. Thomas [17]. The topic of the holomorphically projective $(H P)$ mappings was introduced (for classical, elliptic) Kähler manifolds $\mathbb{K}_{n}^{-}$by T. Otsuki and Y. Tashiro [13], for hyperbolic Kähler manifolds $\mathbb{K}_{n}^{+}$by M. Prvanović [14], and for parabolic Kähler manifolds $\mathbb{K}_{n}^{o}$ by V. V. Vishnevskij [18]. See, e.g., $[1,7,10,11,16,20]$.

Let us mention that geodesic, conformaly geodesic and holomorphically projective mappings were studied under a certain additional condition based on the proportionality of the metrics. It turns out that even under this condition, the mapping is a homothety. See, e. g., [2-6, 8, 12].

In this paper we consider the following question, whether properties of conformal geodesic mappings are applicable for the composition of the conformal and holomorphically projective mappings - conformal holomorphically projective mappings of conformal $e$-Kähler manifolds.

The paper was supported by the project CZ.1.07/2.3.00/30.0035. 
An analysis of the HP mappings of $e$-Kähler manifolds in terms of differentiability is presented in paper by I. Hinterleitner [9]. If the contrary is not specified, consideration is given in the tensor form in the class of real sufficiently smooth functions, the dimension $n \geq 4$, and is not mentioned specially. All the spaces are assumed to be connected.

\section{MAIN PROPERTIES OF KÄHLER AND CONFORMAL KÄHLER MANIFOLDS}

We introduce in the following definition generalizations of (pseudo-) Kähler, conformal Kähler and Hermitian manifolds.

Definition 1. An $n$-dimensional (pseudo-) Riemannian manifold $(M, g)$ is called an $e$-Hermite manifold $H_{n}=(M, g, F)$ if besides the metric tensor $g$, a tensor field $F$ ( $\neq \mathrm{Id}$ ) of type $(1,1)$ is given on manifold $M_{n}$, such that $F^{2}=e \mathrm{Id}, e= \pm 1$, and $g(X, F X)=0$ for all tangent vector $X$. Moreover, if $\nabla F=0$ then $H_{n}$ is $e$-Kähler manifold $K_{n}=(M, g, F)$.

We remark, that for $e=-1$ are manifolds $H_{n}$ and $K_{n}$ (pseudo-) Hermitian and (pseudo-) Kähler manifolds, respectively, $F$ is (almost) complex structure. For $e=$ 1 we get hyperbolic Hermitian and hyperbolic Kähler manifold, respectively, $F$ is (almost) product structure. See $[9,12,12,16,20]$.

We remind the fundamental knowledge of a conformal mapping, that to be found in many monographs, see $[6,7,12,16,20]$.

Definition 2. A diffeomorphism $f$ between pseudo-Riemannian manifolds $V_{n}=$ $(M, g)$ and $\bar{V}_{n}=(\bar{M}, \bar{g})$ is called a conformal mapping, if $f$ preserves angles between all (smooth) curves on $V_{n}(n \geq 2)$.

Equivalently, a mapping $f: V_{n} \rightarrow \bar{V}_{n}$ is conformal if and only if $\bar{g}=\rho \cdot g$, where $\rho$ is a nowhere zero function on $M$ and we will again suppose, $\bar{M}=M$.

From the equation $\bar{g}=\rho \cdot g$ it follows that

$$
(\bar{\nabla}-\nabla)_{X} X=2 \sigma(X) \cdot X-g(X, X) \cdot \Sigma,
$$

where $\nabla$ and $\bar{\nabla}$ are the Levi-Civita connections on $V_{n}$ and $\bar{V}_{n}$, respectively, $\sigma(X)=$ $\frac{1}{2} \nabla_{X} \ln |\rho|, \sigma(X)=g(X, \Sigma)$ and $X$ is an arbitrary tangent vector.

Let us recall a definition of a conformal Weyl tensor $C$ on $V_{n}(n \geq 3)$ :

$$
C_{i j k}^{h}=R_{i j k}^{h}+\delta_{j}^{h} L_{i k}-\delta_{k}^{h} L_{i j}+L_{j}^{h} g_{i k}+L_{k}^{h} g_{i j},
$$

where $L_{i j}=-\frac{1}{n-2}\left(R_{i j}-\frac{R}{2(n-1)} g_{i j}\right), L_{i}^{h}=g^{h \alpha} L_{\alpha i}, R_{i j k}^{h}$ are the components of the Riemannian tensor of $(M, g), R_{i j}=R_{i \alpha j}^{\alpha}$ are the components of the Ricci tensor, $R=R_{\alpha \beta} g^{\alpha \beta}$ is the scalar curvature and $g^{i j}$ are components of the inverse matrix of $g_{i j}$.

If there is a conformal mapping $V_{n} \rightarrow \bar{V}_{n}(n>2)$, then the conformal Weyl tensor remains invariant (i. e. $\bar{C}=C$ ). The converse is not true. 
For $n>3$, a pseudo-Riemannian space is locally conformally flat if and only if, the conformal Weyl tensor vanishes $(C=0)$.

Definition 3. A conformal e-Kähler manifold is conformally equivalent to $e$-Kähler manifold.

Clearly, any conformal $e$-Kähler manifold $K_{n}$ may be considered as an $e$-Hermite manifold and it may be characterised by an $e$-Hermite structure. This structure has the following properties (for $e=-1$, see [15]):

$$
\nabla_{Y} F(X)=\varphi(X) \cdot Y-g(X, Y) \cdot \Phi+\varphi(F X) \cdot(F Y)+g(F X, Y) \cdot F \Phi,
$$

where $\varphi(X)=g(X, \Phi)=\nabla_{X} \mathscr{F}, \mathscr{F}$ is a function on $M$ and $X, Y$ are tangent vector fields.

\section{HOLOMORPHICALLY PROJECTIVE MAPPINGS OF $e$-KÄHLER MANIFOLDS}

Assume the $e$-Kähler manifolds $K_{n}=(M, g, F)$ and $\bar{K}_{n}=(\bar{M}, \bar{g}, \bar{F})$ with metrics $g$ and $\bar{g}$, structures $F$ and $\bar{F}$, the Levi-Civita connections $\nabla$ and $\bar{\nabla}$, respectively. Likewise, as in [13]

Definition 4. A curve $\ell$ in $K_{n}$ which is given by the equation $\ell=\ell(t), \lambda=$ $d \ell / d t(\neq 0), t \in I$, where $t$ is a parameter is called analytically planar, if under the parallel translation along the curve, the tangent vector $\lambda$ belongs to the twodimensional distribution $D=\operatorname{Span}\{\lambda, F \lambda\}$ generated by $\lambda$ and its conjugate $F \lambda$, that is, it satisfies $\nabla_{t} \lambda=a(t) \lambda+b(t) F \lambda$, where $a(t)$ and $b(t)$ are some functions of the parameter $t$.

Particularly, in the case $b(t)=0$, an analytically planar curve is a geodesic.

Definition 5. A diffeomorphism $f: K_{n} \rightarrow \bar{K}_{n}$ is called a holomorphically projective mapping if $f$ maps any analytically planar curve in $K_{n}$ onto an analytically planar curve in $\bar{K}_{n}$.

Let there exist a HP mapping $f: K_{n}=(M, g, F) \rightarrow \bar{K}_{n}=(\bar{M}, \bar{g}, \bar{F})$. Since $f$ is a diffeomorphism, we can suppose local coordinate charts on $M$ or $\bar{M}$, respectively, such that locally $f: K_{n} \rightarrow \bar{K}_{n}$ maps points onto points with the same coordinates, and $\bar{M}=M$.

A manifold $K_{n}$ admits a holomorphically projective mapping onto $\bar{K}_{n}$ if and only if the following equations [12]

$$
\bar{\nabla}_{X} Y=\nabla_{X} Y+\psi(X) \cdot Y+\psi(Y) \cdot X+e \psi(F X) \cdot F Y+e \psi(F Y) \cdot F X
$$

hold for any tangent fields $X, Y$ and where $\psi$ is a differential form. If $\psi \equiv 0$ than $f$ is affine or trivially holomorphically projective. Beside these facts it was proved [12], that $\bar{F}= \pm F$; for this reason we can suppose that $\bar{F}=F$. 
The holomorphically projective tensor, which is defined by the following form,

$$
\begin{aligned}
P_{i j k}^{h}= & R_{i j k}^{h} \\
& +\frac{1}{n+2}\left(\delta_{k}^{h} R_{i j}-\delta_{j}^{h} R_{i k}-e F_{k}^{h} R_{i \alpha} F_{j}^{\alpha}+e F_{j}^{h} R_{i \alpha} F_{k}^{\alpha}+2 e F_{i}^{h} R_{j \alpha} F_{k}^{\alpha}\right)
\end{aligned}
$$

is invariant with respect to holomorphically projective mappings, i. e. $\bar{P}=P$.

It is known, that an $e$-Kähler $K_{n}$ is a manifold of the constant holomorphically projective curvature if and only if the holomorphically projective tensor vanishes $(P=0)$.

\section{CONFORMAL HOLOMORPHICALLY PROJECTIVE MAPPINGS}

After we have sketched some basic properties of holomorphically projective and conformal mappings, let us focus our attention to the already mentioned conformal holomorphically projective ones. In papers $[8,9]$ by I. Hinterleitner so called conformal projective mappings were studied. These mappings are closely related to our subject. Inspired by her observations, we will derive some further results on them.

Definition 6. A diffeomorphism $f: K_{n} \rightarrow \bar{K}_{n}$ is caled a conformal holomorphically projective mapping if $f=f_{1} \circ f_{2} \circ f_{3}$, where

$$
\begin{array}{lll}
f_{1}: & K_{n}=(M, g, F) \rightarrow{ }^{1} K_{n}=\left(M,{ }^{1} g, F\right) & \text { is a conformal mapping, } \\
f_{2}: & { }^{1} K_{n}=\left(M,{ }^{1} g, F\right) \rightarrow{ }^{2} K_{n}=\left(M,{ }^{2} g, F\right) & \text { is a HP mapping and } \\
f_{3}: & { }^{2} K_{n}=\left(M,{ }^{2} g, F\right) \rightarrow \bar{K}_{n}=(M, \bar{g}, F) & \text { is a conformal mapping. }
\end{array}
$$

Evidently, $K_{n}$ and $\bar{K}_{n}$ are conformal $e$-Kähler manifolds, and ${ }^{1} K_{n}$ and ${ }^{2} K_{n}$ are $e$-Kähler manifolds. We will assume, that structures $F$ are on the same manifold $M$. We have the following theorem.

Theorem 1. A diffeomorphism $f: K_{n}=(M, g, F) \rightarrow \bar{K}_{n}=(M, \bar{g}, \bar{F})$ is a conformal holomorphically projective mapping if and only if for each vector field $X$ the following condition holds

$$
(\bar{\nabla}-\nabla)_{X} X=2 \psi(X) \cdot X+e 2 \psi(F X) \cdot F X+g(X, X) \cdot \Sigma+\bar{g}(X, X) \cdot \Omega,
$$

where $\psi$ is a differential 1-form, $\Sigma$ and $\Omega$ are vector fields and there exist the functions $\varrho_{1}, \varrho_{2}$ and $\varrho_{3}$ on the manifold $M$ such that for each field $X$,

$$
\nabla_{X} \varrho_{1}=g(X, \Sigma), \quad \nabla_{X} \varrho_{2}=\bar{g}(X, \Omega), \quad \nabla_{X} \varrho_{3}=\psi(X) .
$$

Proof. The necessity of (4.1) and the existence of the functions $\varrho_{1}, \varrho_{2}$ and $\varrho_{3}$ follow from the relations (2.1) and (3.1). The conditions are sufficient due to the following observation.

Suppose the conditions (4.1) are satisfied. Then one may construct metrics ${ }^{1} g=$ $\exp \left(-2 \varrho_{1}\right) \cdot g$ and ${ }^{2} g=\exp \left(2 \varrho_{2}\right) \cdot \bar{g}$. Computing the difference between the LeviCivita connections associated to ${ }^{1} g$ and ${ }^{2} g$, we get formula, thus according to (3.1), the spaces ${ }^{1} K_{n}$ and ${ }^{2} K_{n}$ are in HP correspondence. 
It is evident that the relation of "being conformal holomorphically projective equivalent" is symmetric and reflexive. Unfortunately, the conformal holomorphically projective mappings do not form a group because of lack of transitivity - the relation is not an equivalence relation.

\section{CONFORMAL HP MAPPINGS WITH INITIAL CONDITIONS}

We generalized the following Theorem:

Theorem 2 (Chudá and Mikeš [4]). Let $f$ be a holomorphically projective mapping between e-Kähler manifolds $(M, g, F)$ and $(M, \bar{g}, \bar{F}), x_{0} \in M$ and $\bar{x}_{0}=$ $f\left(x_{0}\right)$. Suppose that the initial condition $\bar{g}\left(\bar{x}_{0}\right)=k \cdot g\left(x_{0}\right)$ is satisfied for a $k \in \mathbb{R}$. If the holomorphically projective tensor does not vanish at $x_{0}$, then the mapping $f$ provides a homothety between $(M, g, F)$ and $(M, \bar{g}, \bar{F})$, i.e. $\bar{g}=k \cdot g, k=$ const.

We introduce: $Q_{i j k}^{h}=\delta_{j}^{h} g_{i k}-\delta_{k}^{h} g_{i j}-\frac{n-1}{3 e} \cdot\left(F_{j}^{h} F_{i k}-F_{k}^{h} F_{i j}+2 F_{i}^{h} F_{j k}\right)$, where $F_{i k}=g_{i \alpha} F_{k}^{\alpha}$, and we prove the following lemma:

Lemma 1. If $x_{0}$ be a fixed point on e-Kähler manifold $K_{n}$ and $P\left(x_{0}\right)=0$, then at the point $x_{0}$ the formula $C_{h i j k}=\frac{R}{n(n+2)} \cdot Q_{h i j k}$ holds.

Proof. Let at the point $x_{0}$ the holomorphically projective tensor vanishes, i.e. $P_{i j k}^{h}=0$. After contraction of $g^{i j}$ we persuade, that $R_{i j}=\frac{R}{n} \cdot g_{i j}$, and finally we get $C_{h i j k}=\frac{R}{n(n+2)} \cdot Q_{h i j k}$.

Theorem 3. Let $f$ be a conformal holomorphically projective mapping between two conformal e-Kähler manifolds $(M, g, F)$ and $(M, \bar{g}, \bar{F})$. If the metrics are proportional at the point $x_{0}$, i.e. $\bar{g}_{i j}\left(x_{0}\right)=\mu \cdot g_{i j}\left(x_{0}\right), \mu \in \mathbb{R}$ and there exists no $\alpha \in \mathbb{R}$ so that $C_{h i j k}\left(x_{0}\right)=\alpha \cdot Q_{h i j k}\left(x_{0}\right)$, then $f$ is conformal.

Proof. Let $K_{n}$ admit a conformal holomorphically projective mapping $f$ onto $\bar{K}_{n}$ and at the point $x_{0} \in M$, the inequality $C_{h i j k} \neq \alpha \cdot Q_{h i j k}$ holds. Because the mapping $f_{1}: K_{n} \rightarrow{ }^{1} K_{n}$ is a conformal, then for metrics ${ }^{1} g_{i j}=f \cdot g_{i j}$ and for conformal Weyl tensor ${ }^{1} C_{i j k}^{h}=C_{i j k}^{h}$ hold.

Therefore the inequality $C_{h i j k} \neq \alpha \cdot Q_{h i j k}$ from $K_{n}$ has on manifold ${ }^{1} K_{n}$ form: ${ }^{1} C \neq{ }^{1} \alpha \cdot{ }^{1} Q$ Based on the contraposition of Lemma 1 we known, that the holomorphically projective tensor ${ }^{1} P \neq 0$.

Consequently are satisfied the conditions from Theorem 2, the mapping $f_{2}$ : ${ }^{1} K_{n} \rightarrow{ }^{2} K_{n}$ is homothetic. Moreover at the point $x_{0}$ the following assertion holds ${ }^{1} g_{i j}\left(x_{0}\right)=\varrho \cdot{ }^{2} g_{i j}\left(x_{0}\right)$, i. e. mapping $f=f_{1} \circ f_{2} \circ f_{3}$ is conformal.

\section{REFERENCES}

[1] A. V. Aminova, "Projective transformations of pseudo-Riemannian manifolds," J. Math. Sci., New York, vol. 113, no. 3, pp. 367-470, 2003. 
[2] M. Chodorová, H. Chudá, and J. al Lami Raad, "On holomorphically projective mappings onto almost Hermitian spaces,” J. Appl. Math., Bratislava, pp. 667-672, 2011.

[3] H. Chudá, M. Chodorová, and M. Shiha, "On composition of conformal and holomorphically projective mappings between conformally Kählerian spaces," J. Appl. Math., Bratislava, 2012.

[4] H. Chudá and J. Mikeš, "On geodesic mappings with certain initial conditions," Acta Math. Acad. Paedagog. Nyházi (N.S.), vol. 26, no. 2, pp. 337-341, 2010.

[5] H. Chudá and J. Mikeš, "Conformally geodesic mappings satisfying a certain initial condition," Arch. Math., Brno, vol. 47, no. 5, pp. 221-226, 2011.

[6] H. Chudá and J. Mikeš, "On holomorphically projective mappings with certain initial conditions," J. Appl. Math., Bratislava, pp. 673-678, 2011.

[7] L. P. Eisenhart, Non-Riemannian Geometry. Princeton Univ. Press. 1926. Amer. Math. Soc. Colloquium Publications, 2000, vol. 8.

[8] I. Hinterleitner, "Special mappings of equidistant spaces," J. Appl. Math., Bratislava, no. 2, pp. 31-36, 2008.

[9] I. Hinterleitner, "On holomorphically projective mappings of e-Kähler manifolds," Arch. Math., Brno, vol. 48, no. 5, pp. 333-338, 2012.

[10] J. Mikeš, "Geodesic mappings of affine-connected and Riemannian spaces," J. Math. Sci., New York, vol. 78, pp. 311-333, 1996.

[11] J. Mikeš, "Holomorphically projective mappings and their generalizations," J. Math. Sci., New York, vol. 89, pp. 1334-1353, 1998.

[12] J. Mikeš, A. Vanžurová, and I. Hinterleitner, Geodesic mappings and some generalizations. Olomouc: Palacky University Press, 2009.

[13] T. Otsuki and Y. Tashiro, "On curves in Kaehlerian spaces," Math. J. Okayama Univ., vol. 4, pp. 57-78, 1954.

[14] M. Prvanović, "Holomorphically projective transformations in a locally product space," Math. Balk., vol. 1, pp. 195-213, 1971.

[15] Z. Radulović and J. Mikeš, "Geodesic mappings of conformal Kähler spaces," Russian Math. (Iz. VUZ), vol. 38, no. 3, pp. 48-50, 1994.

[16] N. S. Sinyukov, Geodesic mappings of Riemannian spaces. Moscow: Nauka, 1979.

[17] T. Y. Thomas, The differential invariants of generalized spaces. Cambr. Univ. Press, 1934, vol. III.

[18] V. V. Vishnevskij, "Integrable affinor structures and their plural interpretations," J. Math. Sci., New York, vol. 108, no. 2, pp. 151-187, 2002.

[19] H. Weyl, "Zur Infinitesimalgeometrie: Einordnung der projektiven und der konformen Auffassung," Nachrichten der Königlichen Gesellschaft der Wissenschaften zu Göttingen; Mathematischphysikalische Klasse, pp. 99-112, 1921.

[20] K. Yano, Differential geometry on complex and almost complex spaces. Pergamon Press, 1965.

\section{Authors' addresses}

\section{Hana Chudá}

Tomas Bata University in Zlín, Department of Mathematics, Faculty of Applied Informatics, nám. T. G. Masaryka 5555, 76001 Zlín, Czech Republic

E-mail address: chuda@fai.utb.cz

\section{Mohsen Shiha}

University of Homs, Department of Mathematics, Homs, Syria

E-mail address: mohsen_sheha@yahoo.com 